\title{
A Change Management Case Study for Safe Opioid Prescribing and Opioid Use Disorder Treatment
}

\author{
Randi Sokol, MD, MPH, MMedEd, Zev Schuman-Olivier, MD, \\ Maren Batalden, MD, MPH, Laura Sullivan, MD, and \\ Allen F. Shaughnessy, PharmD, MMedEd
}

Background: Given that prescribing practices have contributed to the current opioid epidemic and that primary care clinicians are the largest prescribers of opioids, family physicians must consider the twin goals of safely prescribing opioids for patients with chronic pain while effectively identifying and treating those who have developed opioid use disorder (OUD). However, family physicians may feel constrained by a culture and systems in their offices that do not support achieving these twin goals.

Methods: In a family medicine clinic within a larger academic institution that cares for an underserved, multicultural patient population in the greater Boston area, we provide a case study that illustrates the twin goals of safe opioid prescribing and treating OUD. We used 2 models of change management-Lewin's Three-Step Change Theory and the McKinsey $7 \mathrm{~S}$ Model of Change-as a framework to describe our 5-year process of using cultural and structural elements to support these efforts.

Results: Deliberate use of change management theory to support both safe opioid prescribing and treating patients with OUD over the past 5 years resulted in changes to the practices, people, skills, and infrastructure within our clinic. These changes have demonstrated a sense of stability and sustainability and hence now represent our clinic's current culture.

Conclusion: The Lewin and $7 \mathrm{~S}$ models of change can be helpful guides to creating and maintaining a foundation of office-wide culture and structural support to meet the twin goals of safe opioid prescribing and treating patients with OUD. (J Am Board Fam Med 2020;33:129-137.)

Keywords: Addictive Behavior, Boston, Chronic Pain, Family Physicians, Opioid-Related Disorders, Opioids, Pain Management, Patient-Centered Care, Primary Health Care, Quality Improvement

The opioid epidemic has become a palpable reality across the country. Every day in the United States, more than 40 people die from an overdose involving prescription opioids; since 1999, there have been more than 165,000 deaths from overdoses related to prescription opioids, and overdose deaths related to prescription opioids has now passed

This article was externally peer reviewed.

Submitted 25 June 2019; revised 23 September 2019; accepted 25 September 2019.

From Tufts University School of Medicine, Boston, MA (RS, AFS); Department of Psychiatry, Harvard Medical School, Boston, MA (ZS-O); Harvard Medical School, Boston, MA (MB); Malden Family Medicine Center, Cambridge Health Alliance, Malden, MA (LS, RS, AFS).

Funding: none.

Conflict of interest: none declared.

Corresponding author: Allen F. Shaughnessy, PharmD, MMedEd, Tufts University Family Medicine Residency at Cambridge Health Alliance, 195 Canal Street, Malden, MA 02148 (E-mail: Allen.Shaughnessy@Tufts.edu). deaths from illicit opioids. ${ }^{1,2}$ At the same time, there has been a dramatic increase in prescribing opioids for patients with chronic pain. In 2013, 249 million opioid prescriptions were written by physicians, and primary care clinicians were the largest prescribers. ${ }^{1,2}$ Although prescription opioids may provide some analgesic benefit to patients struggling with chronic pain, $21 \%$ to $29 \%$ of patients receiving chronic opioid treatment will misuse their medications and $8 \%$ to $12 \%$ will eventually develop an opioid use disorder (OUD). ${ }^{3}$

The rampant prescribing of opioids may have resulted from an outdated understanding of pain relief and addiction. It was only recently that clinicians have started to move away from thinking of pain as the "fifth vital sign" that should be assessed and treated at every visit ${ }^{4}$ and from thinking that those treated for chronic pain with opioids could not become addicted. ${ }^{5}$ In addition, only recently has society started 
to shift from viewing illicit drug use as a criminal activity warranting arrest and incarceration to a neurobiological disease warranting a chronic disease management approach ${ }^{6,7}$ and that such an approach is well-suited in a primary care setting rather than siloed in addiction treatment programs. ${ }^{8}$

As family physicians embrace these new paradigms, it will be important to consider, in concert, the twin goals of safely prescribing opioids for patients with chronic pain while effectively identifying and treating those who have developed OUD. However, family physicians may feel constrained in their offices by cultures and systems that do not support the achievement of these twin goals.

For example, physicians attempting to decrease opioid dosing in patients with chronic pain may find it difficult to make this change if treatment approaches and practices are not agreed on and coordinated among prescribers. ${ }^{9}$ Routine monitoring of patients, such as checking urine toxicology tests, ${ }^{9}$ setting up controlled substance agreements, and using the state's prescription drug monitoring program $(\mathrm{PDMP})^{10}$ may also be difficult without adequate support systems in place. ${ }^{11}$

Similarly, physicians attempting to treat patients with OUD may face cultural and system-level barriers that prevent treatment from becoming mainstream. Although more family physicians are receiving waivers to provide medication for OUD (MOUD) in the form of buprenorphine-naloxone $(\mathrm{B} / \mathrm{N})$, one-third never go on to prescribe it, largely due to concerns about having appropriate office, nursing, and administrative support; lacking access to mental health clinicians and addiction specialists; and concerns about being the lone $\mathrm{B} / \mathrm{N}$ provider. $^{11-13}$

Thus, treating patients with chronic pain and patients with OUD requires not only individual physician efforts but also a foundation of office-wide cultural and structural support. About 5 years ago our office faced similar barriers. This article describes a case study of our clinic's deliberate approach using change management theory to support individual clinician efforts in meeting these twin goals. ${ }^{14}$

\section{Methods}

Our institution is an academic health care center that serves as a safety net system for a large urban population across the greater Boston area. As part of its vision and mission statement to care for our patients, our institute has declared treating patients with substance use disorders and mental health comorbidities a high priority. Our family medicine clinic is 1 of its 13 primary care sites. We are located in a medium-sized, multicultural city approximately 8 miles north of Boston, Massachusetts. The clinic serves approximately 23,000 empaneled patients with 17 faculty physicians, 24 residents, 6 physician assistants, and 9 behavioral care clinicians, including 3 primary care-based integrated psychologists. Of all the 13 primary care sites in our organization, our site has the largest number of patients with OUD.

Approximately 5 years ago, we became sensitized to the high use of opioids in our community and the rapidly escalating death rate due to opioid overdose. Although our institution recognized the need to focus on treating patients with addiction and made it a core part of its mission statement, prescribing within our clinic varied widely among physicians; our policies on opioid prescribing and monitoring were not well known or followed; and the state-based $\mathrm{PDMP}^{10}$ was not routinely reviewed before prescribing. We had a minimal curriculum to train residents in pain management or OUD treatment; only 1 core faculty member had been trained to provide $\mathrm{B} / \mathrm{N}$ for patients with OUD, and only 5 patients were receiving treatment.

Recognizing the impact of opioid prescribing in our community, we realized we needed to develop a more intentional approach to safely treat patients prescribed opioid analgesics for chronic pain while also treating patients with OUD. We began our approach to process improvement change using the tenets of Lewin's Three-Step Change Theory ${ }^{15}$ to provide the overall approach. Briefly, this theory suggests that practices within an organization are inherently stable and resistant to change. As a result, change only occurs by (1) "unfreezing," in which clinicians are made aware of the need for change; (2) "changing," in which new systems and expectations for behavior are implemented; and 3) "refreezing," in which the new ways of thinking and doing are institutionalized and, therefore, solidified into new practices.

To begin the unfreezing, a resident led a grand rounds presentation to our entire clinic that served as a call-to-action, informing participants about the current opioid epidemic affecting our community, 
Figure 1. Poster highlighting drug-related deaths in our practice catchment area.

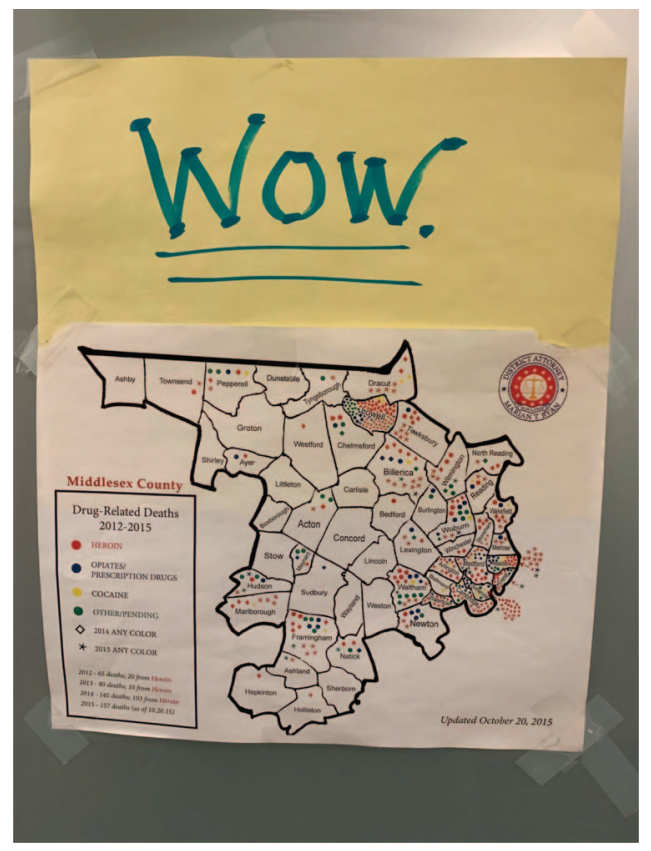

the efficacy of treating OUD with medication, and explaining why this effort should be incorporated into our clinic. At the same time, a relatively new faculty member kept the conversation alive by displaying a poster on her office door highlighting the local drug overdose rates (Figure 1), attracting passersby to pause and consider the issue. Situated within a larger system that had stated its intentions to prioritize addressing addiction and safe opioid prescribing, these efforts launched further clinical and residency attention to formally and systemically examine and identify the gap between current and desired performance.

To guide specific aspects of our change process, we used the principles of the McKinsey 7S model of change ${ }^{16}$ management. Briefly, this model identifies 6 barriers that need to be addressed to support the adoption and maintenance of new practices. The model considers 3 "hard" elements that can be readily seen, including strategy, systems, and structure. It also recognizes 3 "soft" elements that are more intangible, including the types of people, the skills of the people, and the overarching leadership style within the organization. Managing change by addressing all these barriers leads to the $7^{\text {th }}$ "S," creation of shared values underpinning the culture of the group. We used this structure to meet our twin goals of enhancing the care of patients with chronic pain and treating patients with OUD (see Table 1).

\section{Hard Elements}

Strategy: The Plan of Action to Be Used

To promote the safe and appropriate use of opioids, our strategy began by developing shared general principles for safe opioid prescribing and then supporting them through education and health informatics systems. These principles fit into systemwide strategies to address OUD and chronic pain management, although the process began locally and not because of an institutional directive.

To care for patients with OUD, we instituted processes that created a safe and welcoming environment that destigmatized opioid use, greatly expanded use of MOUD primarily through shared medical appointments, ${ }^{17}$ and emphasized a teambased approach to care.

Both strategies were supported by a chronic pain and OUD curriculum for our residents and faculty and training for all members of the patient care team.

\section{Systems: Daily Activities and Procedures Implemented to Get the Job Done}

To improve the management of chronic pain, our clinic created a workgroup comprising faculty and residents to develop guidelines for prescribing opioids based on institution-level approaches and policies around safe opioid prescribing that represented evidence-based and best-practice recommendations. These guidelines were implemented through the use of electronic health record smart-phrases, note templates, and clinical workflows to ensure consistency in application and to foster collaborative care around safe prescribing, monitoring, and response to aberrant patient behavior. These efforts were augmented by the introduction of in-office mental health clinicians and by a consultation service for complex cases.

OUD care within our clinic is supported by institution-wide substance use disorder-related efforts, including at-large addiction nurses who provide central triaging, first-point contact, and ongoing support to patients with OUD. In addition, institution-wide list-serves serve as forums to pose questions and get support from providers across the institution with various experiences in addiction medicine. 


\section{Structure: How Our System Is Organized/Who Reports to Who}

To change our structure of managing patients with chronic pain, we added a quality improvement process that was part of a larger, institution-wide project. We developed a registry of all patients on chronic opioids and a dashboard with clearly defined quality metrics. The clinic director used this registry to review these metrics at regular staff meetings.

We also created a structure to improve the management of patients with OUD by creating an interdisciplinary team comprising a physician, physician assistant, nurse, an appointment scheduler, medical assistant, and psychotherapist to provide group-based opioid treatment (GBOT) as the primary treatment approach to OUD ${ }^{17-19}$ and to provide oversight and support to all providers. All patients diagnosed with OUD were referred to this GBOT team for initial treatment. The team had designated time for weekly meetings to discuss patient care, and a full-time addiction nurse served as the hub of the team, always available to patients and clinicians.

\section{Soft Elements}

\section{Staff: The People and Their Special Capabilities}

For chronic pain management, we use a team-based, patient-centered medical home approach to clinical care, in which each member of the clinical team was taught to manage specific responsibilities when patients are seen for chronic pain visits. ${ }^{20-23}$ This approach also includes utilization of primary care-based, on-site mental health staff who provide individual and group-based treatment for patients with chronic pain. As our institute evolved to dedicate more time and resources to managing chronic pain, our mental health staff (psychologists and care partners) at all 13 primary care sites were trained to offer evidencebased chronic pain treatment modalities.

For management of patients with OUD, we identified a core group of clinical and administrative staff members who expressed an interest and passion to work collaboratively helping patients with OUD. They were given designated time for training and mentorship to build their competencies over time. Both the lead physician and nurse have since acquired specialized addiction training and made addiction care a majority part of their practice. At the larger, system-wide level, our institution hired additional nurse case managers, who had specialized training in addiction to support the integrated health system efforts to provide OUD treatment across all primary care sites, hospitals, and our outpatient addiction services site.

In addition, we created a team comprising a psychologist, psychiatrist, pharmacist, addiction experts, palliative care, and primary care physician to provide consultation services for chronic pain and addiction management.

\section{Skills: Skills and Competencies Needed to Get the Job Done}

In caring for patients with chronic pain, medical assistants were trained to complete the prework of the visit, such as collecting urine samples for toxicology testing, checking the PDMP, and administering questionnaires. Care partners learned how to teach patients about nonpharmacologic approaches to pain management, such as relaxation and stress management techniques, cognitive restructuring, and pacing strategies. Psychologists used specific psychotherapeutic modalities for pain management, including Acceptance Commitment Therapy, Cognitive Behavioral Therapy, and Mindfulness.

In addition to training and certification of all physicians to provide MOUD, all nurses, staff members, and clinicians received training in emergency opioid overdose reversal via naloxone administration. We used regular meetings with all clinical and administrative staff to share personal stories of opioid addiction and overdose as well as patient success stories to create a culture that destigmatizes patients with OUD and promotes a medical treatment model.

\section{Leadership Style}

In managing patients with chronic pain, we used a participative leadership model, ${ }^{24}$ which encourages the participation of all members of the practice with the final decision made by those in official leadership positions. Our clinic director shared institution-based and site-specific recommendations at clinic-wide meetings. She invited discussion and solicited feedback from providers to promote buy-in and create a shared approach to managing patients with chronic pain. Emerging change leaders ${ }^{25}$ bore more responsibility, such as ongoing revision of clinic policy and oversight of provider education.

In managing patients with OUD, we used a centralized leadership approach in which a physician and full-time addiction nurse assumed clinic- 
Strategy: The plan of action to be used.

- Develop shared general principles for safe prescribing of chronic opioids in accordance with expert recommendations that standardizes a team-based, clinic-wide approach

- Develop new curricula for residents and other clinicians in the practice

- Develop support systems among clinicians managing patients on chronic opioids for chronic pain

Systems: Daily activities and procedures instituted to get the job done

- Create, implement, and monitor a policy for prescribing opioids, addressing: initiating prescribing, prescribing to legacy patients

- Develop a workflow to document opioid use on every problem list

- Implement and enforce adherence to controlled substance agreements

- Integrate mental health clinicians

- Create a specialized, interdisciplinary referral service for complex patients with pain and/or addiction

- Create a monthly forum for discussion of difficult and complex patients

- Create a clinic workflow for medical assistants to check the prescription monitoring program, administer the pain functional assessment tool, and collect urine for drug screen monitoring

Develop smart-phrases ("dot-phrases") for our electronic medical record to aid with note writing and clinical decision-making and to aid in adherence to policies

Structure: How our system is organized/who reports to who

- Institution-wide chronic pain working group develops quality improvement metrics around safe prescribing behaviors

- Medical director provides oversight of clinicians managing patients receiving chronic opioids

Style: The style of leadership

- Participatory leadership led by the clinic director

- Charismatic leadership provided by a key opinion leader

Staff: The people and their special capabilities

- Physicians, physician assistants, nurses, medical assistants, care partners (social workers), and primary care health psychologists, each with unique roles to care for patients at the individual level
- Develop team-based care to provide care for patients 6with OUD

- Institute office-wide medication-for OUD treatment with buprenorphine/naloxone and intramuscular (IM) naltrexone

- Foster a safe and welcoming environment that destigmatizes substance use disorders and approaches addiction as a neurobiological disease
- Assure prescribers, preceptors, and residents (after first year) receive onsite buprenorphine waiver training

- Develop and institute group visits (shared medical appointments) for patients with OUD

- Develop a workflow for centralized intake of all new patients for OUD treatment utilizing one full-time addiction nurse

- Create a health system-wide list-serve to share best practices, ask questions, get further support around OUD management

- Develop a referral system to specialized outpatient addiction services for selected patients

- Institute team meeting time to discuss management decisions and revise logistical components of group-based management

\section{A multidisciplinary consultation team with expertise complex pain/addiction problems}

Skills: Skills and competencies needed to get the job done

- Curriculum to disseminate evidence-based guidelines and clinic-specific policies around safe opioid prescribing, nonopioid pharmacologic options, and nonpharmacological approaches

- Care partners training to provide continuity and to teach nonpharmacological approaches to pain management

Medical assistant training in intake and urine collection
- One lead physician and one full-time addiction nurse provide oversight to management of all patients with OUD

- Designated dedicated interdisciplinary team provides group-based opioid treatment (shared medical appointments)

- Centralized clinic interdisciplinary team with top-down leadership from lead physician and lead addiction nurse

- Mentorship for decision-making for patients with OUD is provided by an interdisciplinary care team, a separate consultation service, and a system-wide shared email list-serve for consultation questions

- A family physician obtained board certification in addiction medicine; a nurse completed enhanced addiction training

- Physician assistant and back-up physicians assist with the oversight of managing patients with OUD

- Buprenorphine training for all physicians

- Naloxone administration training to all staff

- Longitudinal addictions curriculum for residents

- Reception staff training in the special needs of patients with OUD 
wide oversight and were further supported by an interdisciplinary care team, a system-wide consultation service, and a shared provider list-serve.

\section{Results}

As a result of these changes implemented over the past 5 years, we were able to unfreeze the existing culture and have since refrozen this culture, with its associated strategy, systems, structure, staff, skills, and leadership style, to care for patients with chronic pain and patients with OUD.

For patients with chronic pain, our efforts resulted in the creation of standardized rules (see Appendix). Our institution developed quality metrics around patients with chronic pain on chronic opioids and electronic health record tracking methods. Among our patients with chronic pain on chronic opioids ( $1 \%$ of our empaneled patients), $73 \%$ have signed a controlled substance use agreement, $60 \%$ have had adequate urine monitoring over the past year, and only 35 patients receive high doses (more than 100 morphine milligram-equivalents per day). Our goal is to continue to improve these measures.

For patients with OUD, our efforts have resulted in policies that require all physicians and residents to receive $\mathrm{B} / \mathrm{N}$ training and waiver. Clinic-wide meetings have resulted in all staff members being trained on responding to an opioid overdose using naloxone. Our group-based approach to treating OUD has become embedded into our clinical practice, with clearly defined roles and responsibilities for all team members (including designation of a full-time addictions nurse). We provide multiple weekly group visit medical appointments on a rolling basis so that patients have received continuous access to care since its inception, and we are able to care for approximately 125 patients at any given point in time. Similar to efforts around chronic pain management, our institution has set OUD metrics and now tracks them via our electronic health record. Thirty percent of patients with a diagnosis of OUD are receiving MOUD, with a goal of $34 \%$.

We provide ongoing, regularly scheduled training and support to providers via monthly practice inquiry meetings ${ }^{26}$ and biweekly interdisciplinary consultation service meetings that support primary care clinicians in managing difficult pain and addiction cases. We have developed and implemented a chronic pain and addiction curriculum for our residents that builds in experiential and didactic learning throughout all 3 years of their training.

\section{Limitations}

The goal of this project was to describe our process for implementing change in an organization, rather than showing its effect. We believe that using the Lewin and McKinsey $7 \mathrm{~S}$ models to guide change can be helpful for practices wishing to address the twin goals of better management of patients with chronic pain with opioid analgesics and the treatment of patients with OUD. There are other models of change management that may also be helpful to provide guidance. ${ }^{27-29}$

Although we feel the culture has changed within our clinic, there are likely pockets within our organization in which the old attitudes remain. Our 5 -year time frame to measure culture change induction and sustainability is relatively arbitrary, although it fits into standard estimates of culture change. ${ }^{30}$ Rather, the nature of the cycle of a change project is inherently iterative and requires constant reinforcement. Our results attempt to highlight the practices, people, skills, and infrastructure that have been built into our system, have demonstrated a sense of stability and sustainability, and now represent our clinic's current culture.

We recognize that, within this overall framework, the change components we chose are unique to our environment; other organizations should choose components that are best suited to their local setting and context. Our environment was likely bolstered by a clinical and educational milieu that supports ongoing change improvement efforts. At the system-wide level, our institute supported change efforts around managing chronic pain and addiction, making it a priority in its vision and mission, providing time and resources to support institution-wide chronic pain and OUD treatment. At the clinic-wide level, we were fortunate enough to have agreement about the need for change from both clinical and residency leadership, an enthusiastic leader who overcame the inertia common in many organizations ${ }^{31}$ to start the change process, and many key leaders and office staff who had received extensive training in organizational change management and process improvement methodology. As a result, continuous quality improvement was an everyday aspect of our practice and change fatigue $^{32}$ was not a barrier. 


\section{Conclusion}

This article describes a case study of an approach to addressing the twin goals of improving opioid prescribing for pain management and treating patients with OUD through a collaborative, interdisciplinary, team-based, evidence-based, and patient-centered culture with associated structural supports. The Lewin and 7S models of change can be helpful approaches to practice transformation because they acknowledge the system, culture, and individuals in an organization that have to be addressed to initiate change and make it stable and sustainable. Other practices interested in such transformation can consider these models as guides.

To see this article online, please go to: http://jabfm.org/content/ 33/1/129.full.

\section{References}

1. 2018 Annual Surveillance Report of Drug-Related Risks and Outcomes - United States. Surveillance Special Report. Washington, DC: Centers for Disease Control and Prevention, U.S. Department of Health and Human Services; 2018. (Accessed June 10, 2019, at https://www.cdc.gov/drugoverdose/pdf/pubs/2018cdc-drug-surveillance-report.pdf).

2. Chen JH, Humphreys K, Shah NH, Lembke A. Distribution of opioids by different types of Medicare prescribers. JAMA Intern Med 2016;176: 259-61.

3. Vowles KE, McEntee ML, Julnes PS, Frohe T, Ney JP, van der Goes DN. Rates of opioid misuse, abuse, and addiction in chronic pain: a systematic review and data synthesis. Pain 2015;156:569-76.

4. Anson P. AMA drops pain as vital sign. Available from: https://www.painnewsnetwork.org/stories/ 2016/6/16/ama-drops-pain-as-vital-sign. Published June 16, 2016.

5. Porter J, Jick H. Addiction rare in patients treated with narcotics. N Engl J Med 1980;302:123.

6. Leshner AI. Addiction is a brain disease, and it matters. Science 1997;278:45-7.

7. Volkow ND, Koob GF, McLellan AT. Neurobiologic advances from the brain disease model of addiction. N Engl J Med 2016;374:363-71.

8. Compton WM, Blanco C, Wargo EM. Integrating addiction services into general medicine. JAMA 2015;314:2401-2.

9. Dowell D, Haegerich TM, Chou R. CDC guideline for prescribing opioids for chronic pain-United States, 2016. MMWR Recomm Rep 2016;65:1-49.

10. Mass.gov. Massachusetts Prescription Awareness Tool (MassPAT). Available from: https://www.mass. gov/guides/massachusetts-prescription-awarenesstool-masspat. Published 2019. Accessed June 10, 2019.
11. Huhn AS, Dunn KE. Why aren't physicians prescribing more buprenorphine? J Subst Abuse Treat 2017;78:1-7.

12. Miotto K, Hillhouse M, Donovick R, et al. Comparison of buprenorphine treatment for opioid dependence in 3 settings. J Addict Med 2012;6:68-76.

13. Hutchinson E, Catlin M, Andrilla CH, Baldwin LM, Rosenblatt RA. Barriers to primary care physicians prescribing buprenorphine. Ann Fam Med 2014;12: 128-33.

14. Nelson EC. Value by design: developing clinical microsystems to achieve organizational excellence, 1st ed. San Francisco: Jossey-Bass; 2011.

15. Burnes B. Kurt Lewin and the planned approach to change: a re-appraisal. J Manag Stud 2004;41:9771002 .

16. Peters TJ, Waterman J, Robert H. In search of excellence. New York: Harper Collins; 2006.

17. Sokol R, Albanese C, Chaponis D, et al. Why use group visits for opioid use disorder treatment in primary care? A patient-centered qualitative study. Subst Abus 2018:39:52-58.

18. Sokol R, LaVertu AE, Morrill D, Albanese C, Schuman-Olivier Z. Group-based treatment of opioid use disorder with buprenorphine: a systematic review. J Subst Abuse Treat 2018;84:78-87.

19. Sokol R, Albanese M, Albanese C, et al. Implementing group visits for opioid use disorder: a case series. Subst Abus 2019;1-7.

20. American Academy of Family Physicians (AAFP), American Academy of Pediatrics (AAP), American College of Physicians (ACP), American Osteopathic Association (AOA). Joint Principles of the PatientCentered Medical Home 2007. Available from: https://www.aafp.org/dam/AAFP/documents/ practice_management/pcmh/initiatives/PCMHJoint. pdf. Published 2007. Accessed September 20, 2019.

21. Lyon RK, Slawson J. An organized approach to chronic disease care. Fam Pract Manag 2011;18:27-31.

22. Putting group visits Into practice. A practical overview to preparation, implementation, and maintenance of group visits at Massachusetts General Hospital. 2012. Available from: https:/www.sgim.org/ File\%20Library/SGIM/Meetings/Annual\%20 Meeting/Meetign \%20Content/AM\%2 014\%20 handouts/WE04-STEPHANIE-EISENSTAT.pdf. Published 2012. Accessed June 10, 2019.

23. Safford BH, Manning C. Six characteristics of effective practice teams. Fam Pract Manag 2012; 19:26-30.

24. Vroom VH, Yetton PW. Leadership and decisionmaking. Pittsburgh, PA: University of Pittsburgh Press; 1973.

25. Sismondo S. How to make opinion leaders and influence people. CMAJ 2015;187:759-760.

26. Sommers LS, Morgan L, Johnson L, Yatabe K. Practice inquiry: clinical uncertainty as a focus for 
small-group learning and practice improvement. J Gen Intern Med 2007;22:246-52.

27. Rogers EM. Diffusion of innovations, 3rd ed. New York, NY: The Free Press; London: Collier Macmillan; 1983.

28. Kotter JP. Leading change. Boston: Harvard Business Review Press; 2012.

29. Thaler RH, Sunstein CR. Nudge: improving decisions about health, wealth, and happiness. New York: Penguin Books; 2009.
30. Kotter JP, Schlesinger LA. Choosing strategies for change. Harv Bus Rev 1979;57:106-14.

31. Collins J. Good to great: why some companies make the leap and others don't. New York: HarperCollins; 2001.

32. Nutting PA, Miller WL, Crabtree BF, Jaen CR, Stewart EE, Stange KC. Initial lessons from the first national demonstration project on practice transformation to a patient-centered medical home. Ann Fam Med 2009;7:254-60. 


\section{Appendix}

Chronic Pain: "The Rules"

1. New patients on opioids from an outside clinic should not be continued since the clinician needs time to review previous records, check MassPAT, and determine if opioids are appropriate

2. Generally, patients on chronic opioids managed by the team should see their primary care clinician a minimum of every third visit

3. Visits should be every 3 months for most patients (they might need to be seen more frequently if actively adjusting meds/higher risk; or space out if low risk)

4. If starting on opioids: use the Opioid Risk Tool to assess risk ${ }^{1}$

5. All patients must have controlled substance agreement; this should be renewed annually

6. Check PDMP for EVERY opioid prescription (document with ".opiateprescriptionadult")

7. Order urine drug screen twice annually for low risk, more frequently for higher risk ${ }^{2}$

\footnotetext{
${ }^{1}$ There are numerous screening tools to assess risk for starting chronic opioids for pain management, including the Opioid Risk Tool (ORT), Diagnosis, Intractability, Risk, Efficacy (DIRE), Screener and Opioid Assessment for Patients with Pain (SOAPP), and Screening Tool for Addiction Risk (START). None of these has been proven superior to the others. (Moore TM, Jones T, Browder JH, et al. A comparison of common screening methods for predicting aberrant drug-related behavior among patients receiving opioids for chronic pain management. Pain Med 2009;10(8): 1426-33. doi: 10.1111/j.1526-4637.2009.00743.x [published Online First: 2009/12/22]) We chose ORT for its simplicity.

${ }^{2}$ Urine drug screen (UDS) frequency should be governed by physician-based judgment and concern for misuse of medications. There is not one established way to risk assess ongoing opioid use but rather numerous ways by using a benefit versus risk framework. Benefit can be assessed using functional scores such as the PEG functional questionnaire that we use and achievement of goals set by the patient and provider. Risk/harm of the opioid medication can be assessed using monitoring in the form of UDS (scheduled or random), pill counts, (scheduled or random), PDMP, reported history, and the Current Opioid Misuse Measure
}

8. Order in Epic: “Addiction” Maintenance/ Pain Management (makes sure that fentanyl, methadone, and buprenorphine are included)

9. Add Chronic Pain Plan in the problem list (“.painplan")-this should be a shared document that is continually updated for care coordination/management between multiple clinicians and inbox coverage

10. Use templated notes: Chronic pain note (".chronicpaininitial" or ".chronicpainfollowup")

11. $\mathrm{PEG}^{3}$ score at every visit (Embedded in note and can use ".painpeg" as a way to monitor pain and quality of life)

12. Goal is to keep daily morphine-equivalents to $<100 \mathrm{mg} /$ day $^{4}$

13. Avoid concurrent central nervous system depressants, especially benzodiazepines

14. Focus on function/quality of life/safety

15. Naloxone prescription; Naloxone instructions in AVS ("naloxone")

16. Refer complex cases to PASS (Pain and Addiction Support Services) Team

(COMM-9) tool. (McCaffrey SA, Black RA, Villapiano AJ, et al. Development of a Brief Version of the Current Opioid Misuse Measure (COMM): The COMM-9. Pain Med 2019; 20(1):113-18. doi: 10.1093/pm/pnx311 [published Online First: 2017/12/14]) Providers are encouraged to use multiple approaches to make an overall benefit versus harm assessment. The Centers for Disease Control and Prevention recommends performing UDS annually (2018 Annual Surveillance Report of Drug-Related Risks and Outcomes United States. Surveillance Special Report.: Centers for Disease Control and Prevention, U.S. Department of Health and Human Services; 2018 [Available from: https://www.cdc.gov/drugoverdose/pdf/pubs/2018-cdc-drug-surveillancereport.pdf accessed June 10, 2019 2019.), and our institute recommends at least every 6 months. For patients at higher risk, based on other benefit versus harm assessments, the physician may decide to do more frequent monitoring; our clinic-working group suggested every 1-3 months for highrisk patients.

${ }^{3}$ https://www.ncbi.nlm.nih.gov/pmc/articles/PMC2686775/.

${ }^{4}$ The Centers for Disease Control and Prevention now suggests less than 90 morphine equivalents/day. 